CMCL 2020

\title{
The Workshop on Cognitive Modeling and Computational Linguistics
}

\section{Proceedings of the Workshop}

November 19, 2020

Online event 


\section{ㄴ) \\ PAROR 709 . CNAS - AMU \\ LANGAGE}

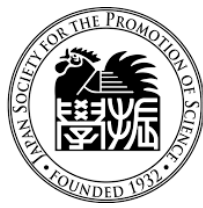

(c) 2020 The Association for Computational Linguistics

Order copies of this and other ACL proceedings from:

Association for Computational Linguistics (ACL)

209 N. Eighth Street

Stroudsburg, PA 18360

USA

Tel: +1-570-476-8006

Fax: +1-570-476-0860

acleaclweb.org

ISBN 978-1-952148-68-2 


\section{Introduction}

Welcome to the Workshop on Cognitive Modeling and Computational Linguistics (CMCL)!

Since the early editions, CMCL has been a reference point for the research at the intersection between Computational Linguistics and Cognitive Science. The edition of this year, because of the COVID-19 pandemic, will not be held in Punta Cana as we originally planned, but it will be the first to be held entirely online. Despite this, we still received 17 regular paper submissions and 10 were accepted for publication (the total acceptance rate is 58\%, the same of the 2019 edition), proving that CMCL is followed by a strong and active research community. We also received 2 cross-submissions, and we accepted one for presentation at the workshop.

The program of this year is rich, stimulating and diversified, with topics ranging from language acquisition to models of linguistic alignment and language disorders. We would like to deeply thank all the reviewers of our PC, without whom we would not have been able to select the best papers for our program.

We also thank Prof Suzanne Stevenson and Prof Richard Futrell, for having accepted our invitation to deliver the keynote speeches.

Finally, thanks to our sponsors, we were able to offer registration fee waivers to $\mathrm{PhD}$ students and to offset the costs of participation of the invited speakers: we gratefully acknowledge support from the Laboratoire Parole et Langage (LPL), Aix-en-Provence (France) and from the Japan Society for the Promotion of Science. 



\section{Organizers:}

Emmanuele Chersoni (Hong Kong Polytechnic University)

Cassandra Jacobs (University of Wisconsin)

Yohei Oseki (University of Tokyo)

Laurent Prévot (Aix-Marseille University)

Enrico Santus (Bayer)

\section{Program Committee:}

Laura Aina (Pompeu Fabre University of Barcelona)

Raquel Garrido Alhama (Max Planck Institute for Psycholinguistics)

Afra Alishahi (Tilburg University)

Philippe Blache (Aix-Marseille University)

Christos Christodoulopoulos (Amazon)

Aniello De Santo (University of Utah)

Micha Elsner (Ohio State University)

Raquel Fernandez (University of Amsterdam)

Abdellah Fourtassi (Aix-Marseille University)

Thomas Francois (Catholic University of Louvain)

Robert Frank (Yale University)

John Hale (University of Georgia)

Anna Ivanova (MIT)

Yu-Yin Hsu (Hong Kong Polytechnic University)

Tim Hunter (UCLA)

Samar Husain (IIT Delhi)

Shalom Lappin (University of Gothenburg)

Gianluca Lebani (University Ca' Foscari Venezia)

Fred Mailhot (DialPad)

Syrielle Montariol (University of Paris Sud - LIMSI CNRS)

Karl Neergaard (University of Macau)

Stephen Politzer-Ahles (Hong Kong Polytechnic University)

Vito Pirrelli (ILC-CNR Pisa)

Carlos Ramisch (Aix-Marseille University)

Giulia Rambelli (University of Pisa)

Roi Reichart (Technion - Israel Institute of Technology)

Rachel A Ryskin (MIT)

William Schuler (Ohio State University)

Marina Sedinkina (University of Munich)

Olga Seminck (Catholic University of Louvain)

Marco Silvio Giuseppe Senaldi (McGill University)

Cory Shain (Ohio State University)

Aaron Steven White (University of Rochester)

Victoria Yaneva (University of Wolverhampton)

Yao Yao (Hong Kong Polytechnic University)

Frances Yung (Saarland University) 
Richard Futrell (University of California Irvine)

Suzanne Stevenson (University of Toronto) 


\section{Table of Contents}

What Determines the Order of Verbal Dependents in Hindi? Effects of Efficiency in Comprehension and Production

Kartik Sharma, Richard Futrell and Samar Husain . . .

Images and Imagination: Automated Analysis of Priming Effects Related to Autism Spectrum Disorder and Developmental Language Disorder

Michaela Regneri, Diane King, Fahreen Walji and Olympia Palikara

Production-based Cognitive Models as a Test Suite for Reinforcement Learning Algorithms

Adrian Brasoveanu and Jakub Dotlacil ................................... 28

Evaluating Word Embeddings for Language Acquisition

Raquel G. Alhama, Caroline Rowland and Evan Kidd ...

Guessing the Age of Acquisition of Italian Lemmas through Linear Regression

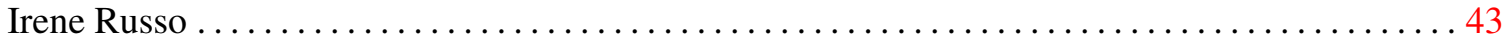

Word Co-occurrence in Child-directed Speech Predicts Children's Free Word Associations

Abdellah Fourtassi . . .

Development of Multi-level Linguistic Alignment in Child-adult Conversations

Thomas Misiek, Benoit Favre and Abdellah Fourtassi ........................... 54

Conditioning, but on Which Distribution? Grammatical Gender in German Plural Inflection

Kate McCurdy, Adam Lopez and Sharon Goldwater.............................. 59

Learning Pronoun Case from Distributional Cues: Flexible Frames for Case Acquisition

Xiaomeng Ma, Martin Chodorow and Virginia Valian ........................... 66

Probabilistic Predictions of People Perusing: Evaluating Metrics of Language Model Performance for Psycholinguistic Modeling

Yiding Hao, Simon Mendelsohn, Rachel Sterneck, Randi Martinez and Robert Frank ......... 75 



\section{Conference Program}

November 19, 2020

\section{9:30-9:45 Introduction}

\section{9:45-10:45 Keynote Talk}

How Languages Carve Up the World: Cognitive Explanation through Computational Modeling

Suzanne Stevenson

\section{0:45-11:15 Break}

\section{1:15-12:15 Session 1: Oral Presentations}

What Determines the Order of Verbal Dependents in Hindi? Effects of Efficiency in Comprehension and Production

Kartik Sharma, Richard Futrell and Samar Husain

Images and Imagination: Automated Analysis of Priming Effects Related to Autism Spectrum Disorder and Developmental Language Disorder

Michaela Regneri, Diane King, Fahreen Walji and Olympia Palikara

\section{2:15-13:30 Lunch Break}




\section{November 19, 2020 (continued)}

\section{3:30-13:45 Poster Booster}

\section{3:45-14:45 Poster Session}

Production-based Cognitive Models as a Test Suite for Reinforcement Learning Algorithms

Adrian Brasoveanu and Jakub Dotlacil

Evaluating Word Embeddings for Language Acquisition

Raquel G. Alhama, Caroline Rowland and Evan Kidd

Guessing the Age of Acquisition of Italian Lemmas through Linear Regression Irene Russo

Word Co-occurrence in Child-directed Speech Predicts Children's Free Word Associations

Abdellah Fourtassi

Development of Multi-level Linguistic Alignment in Child-adult Conversations Thomas Misiek, Benoit Favre and Abdellah Fourtassi

Conditioning, but on Which Distribution? Grammatical Gender in German Plural Inflection

Kate McCurdy, Adam Lopez and Sharon Goldwater

Probabilistic Weighting of Perspectives in Dyadic Communication (crosssubmission)

Rachel A Ryskin, Suzanne Stevenson, Daphna Heller

Pressures on Language Emergence: Least Effort, Object Constancy and Frequency (Findings of EMNLP)

Diana Rodriguez Luna, Edoardo Maria Ponti, Dieuwke Hupkes, Elia Bruni

Inferring Symmetry in Natural Language (Findings of EMNLP)

Chelsea Tanchip, Lei Yu, Aotao Xu, Yang Xu 


\section{November 19, 2020 (continued)}

\section{4:45-15:45 Session 2: Oral Presentations}

Learning Pronoun Case from Distributional Cues: Flexible Frames for Case Acquisition

Xiaomeng Ma, Martin Chodorow and Virginia Valian

Probabilistic Predictions of People Perusing: Evaluating Metrics of Language Model Performance for Psycholinguistic Modeling

Yiding Hao, Simon Mendelsohn, Rachel Sterneck, Randi Martinez and Robert Frank

\section{5:45-16:45 Keynote Talk}

Information Processing, Communication, and Word Order Richard Futrell

\section{6:45-17:00 Closing Remarks}


